\title{
Prognostic significance of red blood cell distribution width in gastrointestinal cancers
}

\section{A meta-analysis}

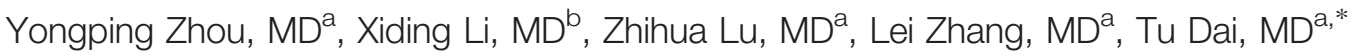

\begin{abstract}
Background: Many publications showed red blood cell distribution width (RDW) might associate with the prognosis of gastrointestinal (Gl) cancers, however, the agreement has not been reached because of controversial results. This meta-analysis aimed to explore the prognostic value of RDW in Gl cancers.

Methods: Four common databases were comprehensively searched to look for relevant studies. The meta-analyses for overall survival (OS) and disease-free survival were performed using hazard ratio (HR) and 95\% confidence interval (Cl), and the metaanalyses for clinical parameters were conducted using odd ratio and 95\% $\mathrm{Cl}$.

Results: A total of 13 studies involving with 3,509 patients with Gl cancers were included into this study. The results showed, compared to patients with low RDW, patients with high RDW tended to have shorter OS $(\mathrm{HR}=1.75,95 \% \mathrm{Cl}=1.57-1.94, P<.01)$ and disease-free survival $(\mathrm{HR}=1.67,95 \% \mathrm{Cl}=1.39-2.00, P<.01)$. High $\mathrm{RDW}$ was associated with larger tumor size $(P<.01)$, worse differentiation $(P=.02)$, deeper invasion $(P<.01)$, earlier lymph node metastasis $(P<.01)$, more advanced clinical stage $(P<.01)$ and higher carcinoembryonic antigen level $(P<.01)$ when compared to low RDW.

Conclusion: High RDW was significantly associated with worse prognosis of Gl cancers, which could be regarded as a prognostic biomarker for Gl cancers. More prospective studies with large sample size and long follow-up period should be carried out to determine the prognostic significance of RDW in Gl cancers in future.

Abbreviations: $\mathrm{Cl}=$ confidence interval, $\mathrm{CSS}=$ cancer-specific survival, $\mathrm{DFS}=$ disease-free survival, $\mathrm{Gl}=$ gastrointestinal, $\mathrm{HR}=$ hazard ratio, NOS $=$ Newcastle-Ottawa scale, OS = overall survival, PFS = progression-free survival, RDW $=$ red blood cell
\end{abstract} distribution width.

Keywords: disease-free survival, gastrointestinal cancer, meta-analysis, overall survival, prognosis, red blood cell distribution width

\section{Editor: Jianxun Ding}

$Y Z$ and $X L$ contributed equally to this research.

Ethics approval and consent to participate was not applicable.

Consent for publication was not applicable.

Please contact author for data requests.

This study was supported by the Health Research Projects of Wuxi Health and Family Planning Commission (Q201806).

The authors have no conflicts of interest to disclose.

Supplemental Digital Content is available for this article.

${ }^{a}$ Department of Hepatobiliary, ${ }^{b}$ Department of General Surgery, Wuxi Second Hospital, Nanjing Medical University, Wuxi, China.

* Correspondence: Tu Dai, Department of Hepatobiliary, Wuxi Second Hospital, Nanjing Medical University No.68 Zhongshan Road, Wuxi 214002, China (e-mail: zyp19840527@163.com).

Copyright @ 2020 the Author(s). Published by Wolters Kluwer Health, Inc. This is an open access article distributed under the terms of the Creative Commons Attribution-Non Commercial License 4.0 (CCBY-NC), where it is permissible to download, share, remix, transform, and buildup the work provided it is properly cited. The work cannot be used commercially without permission from the journal.

How to cite this article: Zhou Y, Li X, Lu Z, Zhang L, Dai T. Prognostic significance of red blood cell distribution width in gastrointestinal cancers: a meta-analysis. Medicine 2020;99:16(e19588).

Received: 10 September 2019 / Received in final form: 20 December 2019 / Accepted: 19 February 2020

http://dx.doi.org/10.1097/MD.0000000000019588

\section{Introduction}

Gastrointestinal (GI) cancers are the most common types of human cancers and have become a critical public problem worldwide. ${ }^{[1,2]}$ Despite great improvements in cancer diagnosis and treatment, approaches to distinguish tumor progression and prognosis of GI cancers still need to be improved. ${ }^{[3,4]}$ A growing number of researchers try to seek promising biomarkers to predict the prognosis of GI cancers. ${ }^{[5-8]}$ However, up to now, no optimal biomarker with satisfactory specificity and sensitivity has been identified.

Systemic inflammatory response has been proved to play a crucial role in cancer progression. ${ }^{[9-11]}$ Previous studies have demonstrated several inflammatory indexes could predict the prognosis of GI cancers, such as platelet-to-lymphocyte ratio, ${ }^{[6,8]}$ neutrophil-to-lymphocyte ratio ${ }^{[12]}$ and lymphocyte-to-monocyte ratio. ${ }^{[13]}$ Recently, several studies have shown that red blood cell distribution width (RDW) was associated with the prognosis of GI cancers. ${ }^{[14-26]}$ However, consensus on the prognostic significance of RDW in GI cancers has not been reached based on the current evidence because of the small sample size and contradictory results. ${ }^{[16,20,21,24,25]}$ Therefore, this meta-analysis was performed to explore the prognostic significance of RDW in GI cancers. 


\section{Materials and methods}

\subsection{Literature search and selection}

The institutional review board of our hospital has approved this study. PubMed, Web of Science, Embase and Cochrane database were comprehensively searched on Jun 5th, 2018. The subject terms and literature search were as follows: "red blood cell distribution width" OR "red cell distribution width" OR "RDW") AND ("cancer” OR “tumor” OR “neoplasm” OR "carcinoma") AND ("prognosis" OR "predict" OR "predictive" OR "survival"). There was no restriction on the language. The references of retrieved articles were also carefully checked to seek more relevant studies. The literature search and selection were completed by 2 authors independently. Any disagreement during the process would be solved by group discussion.

\subsection{Inclusion and exclusion criteria}

The inclusion criteria were as follows:

(1) patients were diagnosed with 1 type GI cancer;

(2) pretreatment RDW was collected;

(3) patients were divided into two groups based on the cut-off value of RDW (high RDW group and low RDW group);

(4) Data were enough to be extracted, such as overall survival (OS), cancer-specific survival (CSS), disease-free survival (DFS), progression-free survival (PFS) or clinical parameters;

(5) full-text was provided to evaluate the quality. The exclusion criteria were as follows: duplicated publications or patients, reviews, comments, case reports, letters, animal experiments, cell experiments or inefficient data.

\subsection{Data extraction and quality assessment}

For each included study, following information was extracted: the first author, country, sample size, gender, high or low level of RDW, cut-off value of RDW, type of GI cancer, outcomes and analysis model of OS. For prognostic items, such as OS, CSS, DFS and PFS, hazard ratio (HR) and corresponding 95\% confidence interval (CI) were directly collected from published articles or indirectly obtained as described by Tierney et al study. ${ }^{[27]}$ The quality of each study was assessed using Newcastle-Ottawa Scale (NOS). ${ }^{[28]}$ This scale contained three domains: selection of participants, comparability of study groups, and the ascertainment of outcomes of interest. Study with NOS $\geq 7$ was considered to be with relatively high quality. ${ }^{[28]}$

\subsection{Statistical analysis}

All analysis in this study was conducted with Review Manager 5.3 (Nordic Cochrane Center) and Stata 12.0 software (Stata, College Station, TX). For prognostic variables (eg, OS, CSS, DFS, and PFS), HR and corresponding 95\% CI were utilized as the summary measures. For clinical parameters, such as gender, tumor differentiation and lymph node metastasis, odds ratio and corresponding $95 \%$ CI were used to detect the overall effects. Inter-study heterogeneity was assessed using Chi-square test and $I^{2}$ statistic. A fixed-effect model should be utilized when $I^{2} \leq 50 \%$ or $P>$.10. Inversely, a random-effect model should be applied on account of the obvious heterogeneity $\left(I^{2}>50 \%\right.$ or $\left.P<.10\right)$. Forest plot was generated to detect the association between RDW and prognosis of GI cancers. Funnel plot and Begg test were conducted to assess the publication bias. Sensitivity analysis was conducted to confirm the robustness of results and Galbraith plot was used to detect the source of heterogeneity. The relationship between cut-off value of RDW and HR of OS was explored by Spearman analysis. $P$ values $<.05$ were considered statistically significant.

\section{Results}

\subsection{Literature search and selection}

As shown in Figure 1, a total of 573 articles were initially retrieved. After removal of duplicates, 285 articles remained for further evaluation. Then, 258 articles were directly excluded by scanning titles or abstracts. As for remaining 27 articles, 7 articles were excluded for not focusing on this topic, 5 articles for reviews or comments and 2 articles for insufficient data. At last, 13 studies containing 3509 patients with GI cancers were included into this meta-analysis. ${ }^{[14-26]}$

\subsection{Characteristics of included studies}

The basic information of included studies was showed in Table 1. There were 2551 males and 958 females. Ten studies were conducted in China, ${ }^{[14,15,17,19,20,22-26]} 1$ study was conducted in Japan, ${ }^{[16]} 1$ study was conducted in Italy ${ }^{[18]}$ and 1 study was conducted in Turkey. ${ }^{[21]}$ Except for Sun et al study ${ }^{[19]}$ and Zhang et al study, ${ }^{[22]}$ there were 1150 patients in high RDW group and 1529 patients in low RDW group. Twelve studies reported cutoff value in the form of variation coefficient of RDW, ranged from $12.2 \%$ to $16.0 \% .^{[14,15,17-26]}$ The cut-off value of RDW was 50 in Hirahara et al study ${ }^{[16]}$ in the form of standard deviation of RDW. Five types of cancer were investigated, including esophageal cancer, ${ }^{[14,16,19,20,22]}$ gastric cancer, ${ }^{[15,21,25]}$ hepatocellular carcinoma, ${ }^{[18,24]}$ colorectal cancer $^{[23,26]}$ and hilar cholangiocarcinoma. ${ }^{[17]}$ With respect to outcomes, 2 studies reported CSS, ${ }^{[14,16]} 11$ studies reported OS, ${ }^{[15,17-26]} 9$ studies reported clinical parameters, ${ }^{[14-17,20,21,23,24,26]} 6$ studies reported DFS $^{[15,20,22-24,26]}$ and 1 study reported PFS. ${ }^{[25]}$ Eight studies used multivariate analysis ${ }^{[14,16,17,20,22-24,26]}$ and 5 studies used univariate analysis. ${ }^{[15,18,19,21,25]}$ All included studies had relatively high quality with $\mathrm{NOS} \geq 7^{[14-26]}$

\subsection{Meta-analysis for the association between RDW and OS}

Eleven studies covered $\mathrm{OS}^{[15,17-26]}$ and 2 studies $^{[14,16]}$ covered CSS, all of them were included into the meta-analysis for the association between RDW and OS. As shown in Figure 2, a fixedeffect model was used $\left(I^{2}=43 \%\right)$, and high RDW was associated with shorter OS compared to low RDW in GI cancers $(\mathrm{HR}=$ $1.75,95 \% \mathrm{CI}=1.57-1.94, P<.01)$. Sensitivity analysis indicated a satisfactory robustness of results (Fig. 3). No obvious publication bias was observed based on the funnel plot (Fig. 4) and Begg test $(P=.51)$ (Supplementary Figure 1, http:// links.lww.com/MD/E65). Galbraith plot showed Smirne et al study ${ }^{[18]}$ was the main source of heterogeneity (Supplementary Figure 2, http://links.lww.com/MD/E66), and the heterogeneity was reduced $\left(\mathrm{HR}=1.62,95 \% \mathrm{CI}=1.45-1.83, P<.01 ; I^{2}=0 \%\right)$ after removal of Smirne et al study ${ }^{[18]}$ (Supplementary Figure 3, http://links.lww.com/MD/E67). 


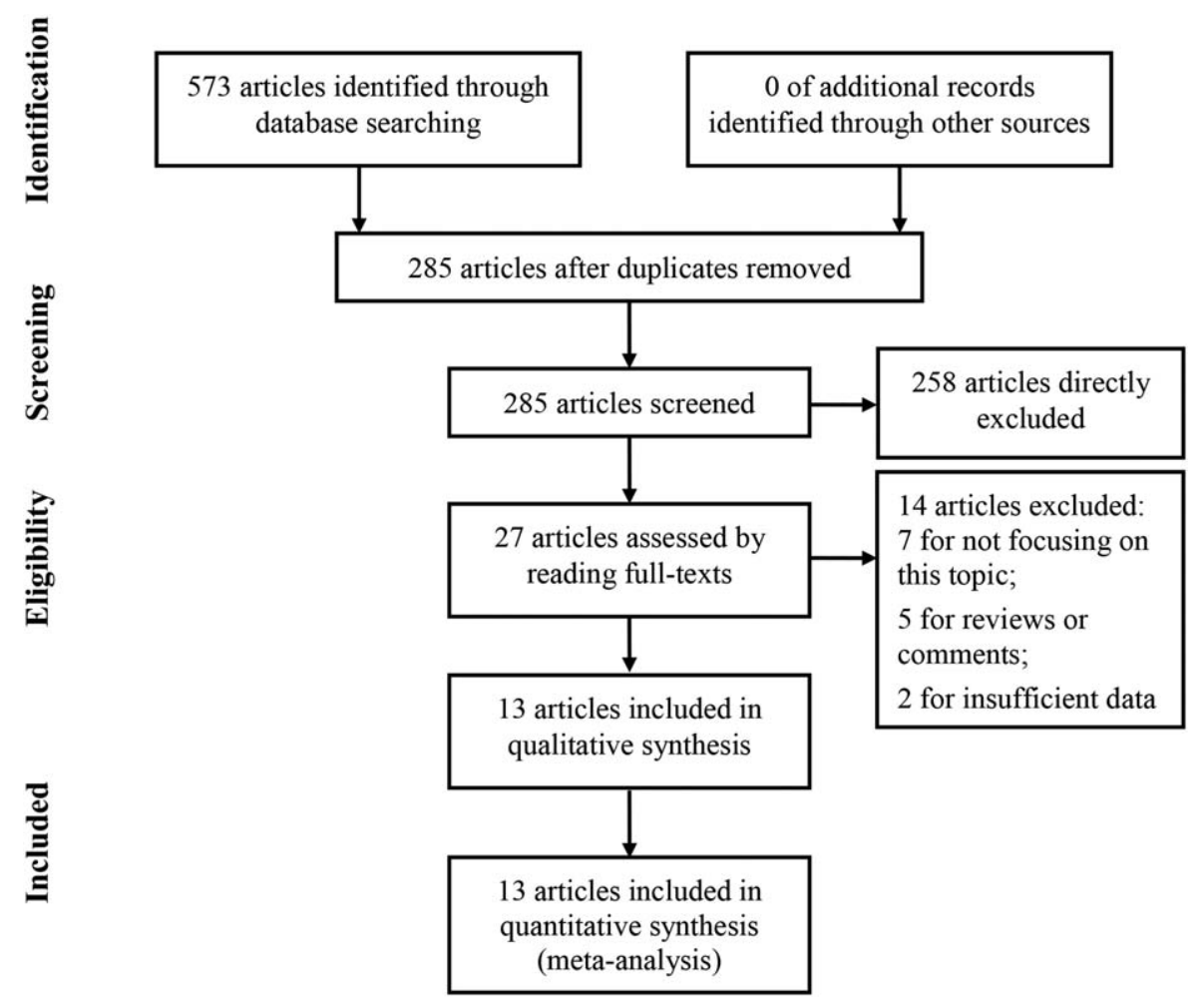

Figure 1. Flow diagram of literature search and selection.

To comprehensively analyze the prognostic value of RDW in GI cancers, subgroup analysis was conducted. Especially, Smirne et al study was not included into the analysis on account of the obviously increased heterogeneity. ${ }^{[18]}$ As listed in Table 2, high RDW was significantly related to worse OS compared to low RDW in GI cancers $(P<.05)$. There was no obvious association between cut-off value of RDW and HR of OS $(P=.56)$ (Fig. 5).

\subsection{Meta-analysis for the association between RDW and DFS}

Six studies reported DFS ${ }^{[15,20,22-24,26]}$ and 1 study reported PFS, ${ }^{[25]}$ all of them were included into the meta-analysis for the association between RDW and DFS (Fig. 6). The results showed, compared to patients with low RDW, patients with high RDW tended to have worse DFS ( $\mathrm{HR}=1.67,95 \% \mathrm{CI}=1.39-2.00$, $\left.P<.01 ; I^{2}=0 \%\right)$. Sensitivity analysis confirmed the robustness of

\section{Table 1}

Characteristics of included studies.

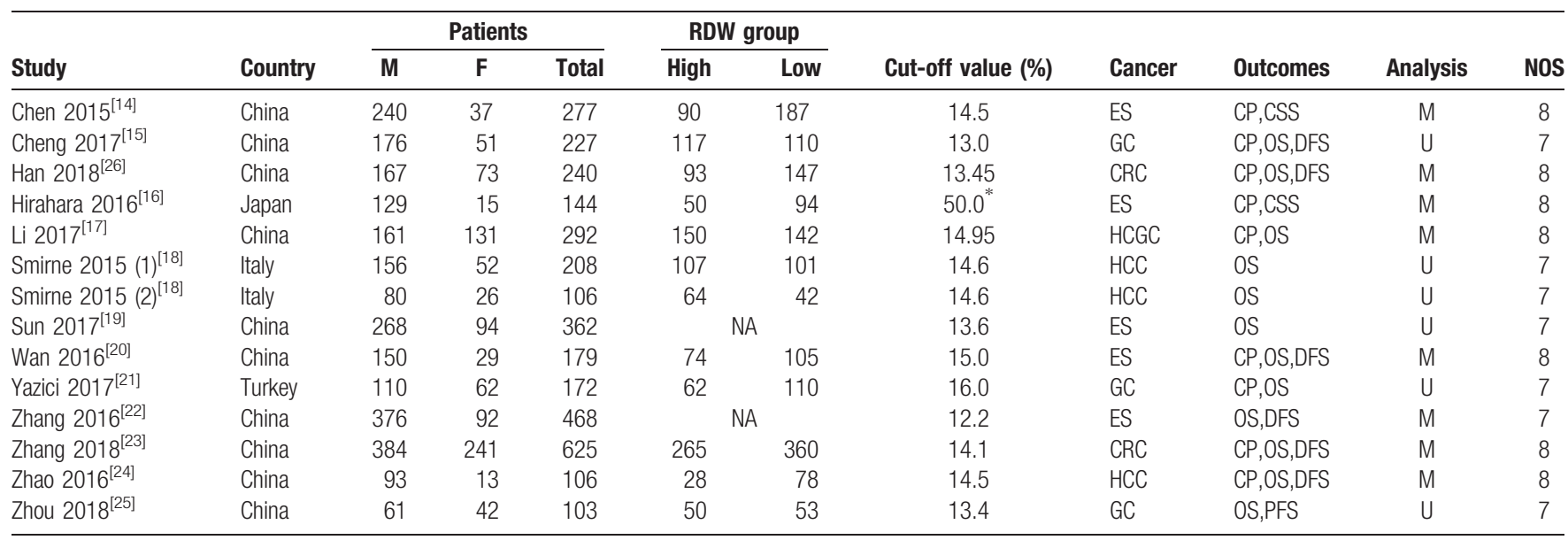

$\mathrm{CP}=$ clinical parameter, $\mathrm{CRC}=$ colorectal cancer, $\mathrm{CSS}=$ cancer-specific survival, $\mathrm{DFS}=$ disease-free survival, $\mathrm{ES}=$ esophageal cancer, $\mathrm{F}=$ female, $\mathrm{GC}=$ gastric cancer, $\mathrm{HCC}=$ hepatocellular carcinoma, $\mathrm{HCGC}=$ hilar cholangiocarcinoma, $\mathrm{M}=$ male, $\mathrm{M}=$ multivariate, $\mathrm{NA}=$ not available, NOS $=$ Newcastle-Ottawa $\mathrm{Scale}, \mathrm{OS}=$ overall survival, $\mathrm{PFS}=$ progression-free survival, $\mathrm{RDW}=$ red blood cell distribution width, $\mathrm{U}=$ univariate.

* The cut-off value in Hirahara 2016 study was reported in the form of standard deviation of RDW. 


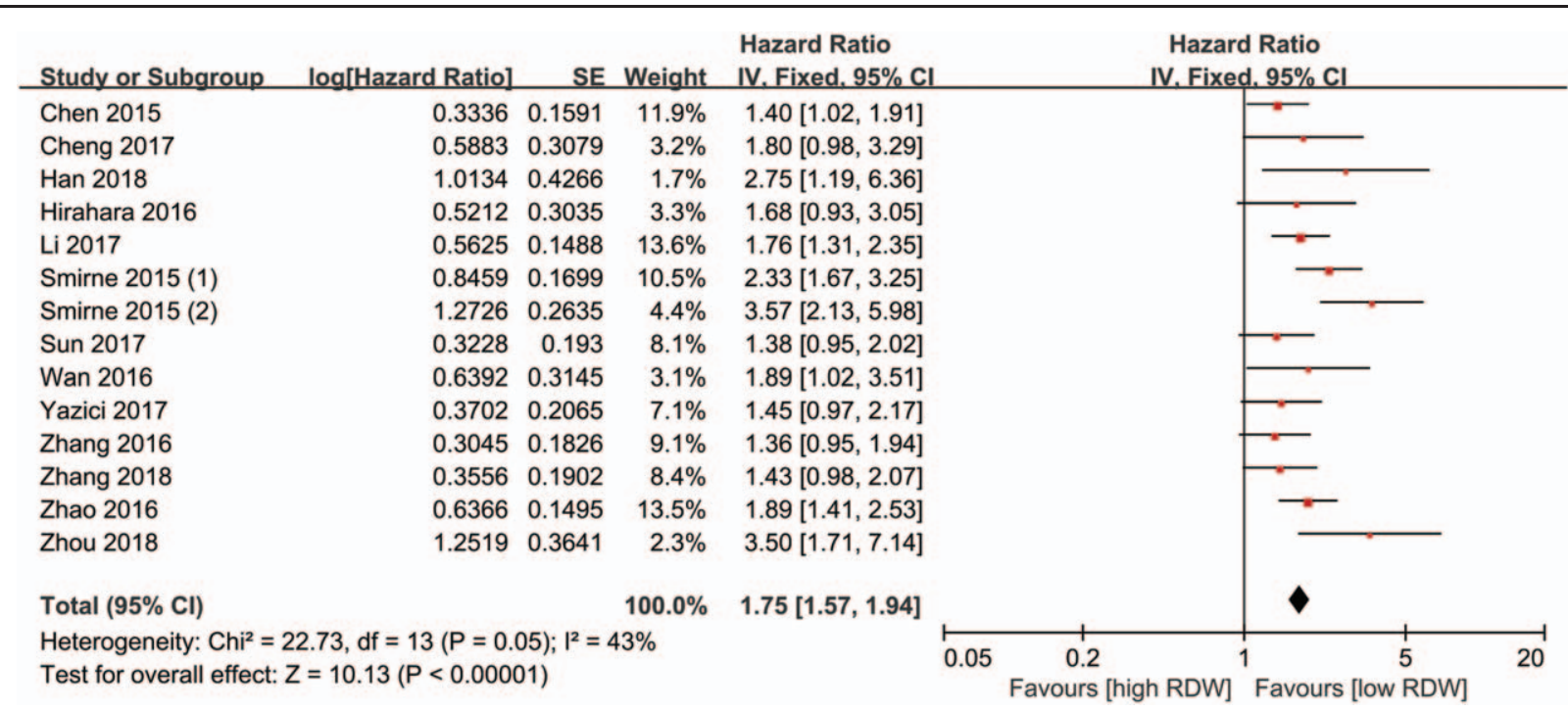

Figure 2. Meta-analysis for the association between RDW and OS. RDW = red blood cell distribution width, OS = overall survival.

the results (Fig. 7). Funnel plot (Fig. 8) and Begg test $(P=.07)$ (Supplementary Figure 4, http://links.lww.com/MD/E68) showed there was no distinct publication bias among included studies.

\subsection{Meta-analysis for the association between RDW and clinical parameters}

As listed in Table 3, there was no obvious relationship between RDW and age $(P=.09)$, gender $(P=.65)$ or vascular invasion $(P=.13)$. However, compared to low RDW, high RDW was significantly associated with larger tumor size $(P<.01)$, worse differentiation $(P=.02)$, deeper invasion $(P<.01)$, earlier lymph node metastasis $(P<.01)$, more advanced clinical stage $(P<.01)$ and higher carcinoembryonic antigen level $(P<.01)$. The funnel plot showed there was no obvious publication bias among included studies (Fig. 9).

\section{Discussion}

Previous investigations showed RDW might have the potential application to predict the prognosis of GI cancers, however, there is no agreement based on current evidence. ${ }^{[15,16,18,20,23,25]}$ In this study, our findings showed high RDW might predict worse OS and DFS in GI cancers. Besides, high RDW was related to larger tumor size, worse differentiation, deeper invasion, earlier lymph node metastasis, more advanced clinical stage and higher

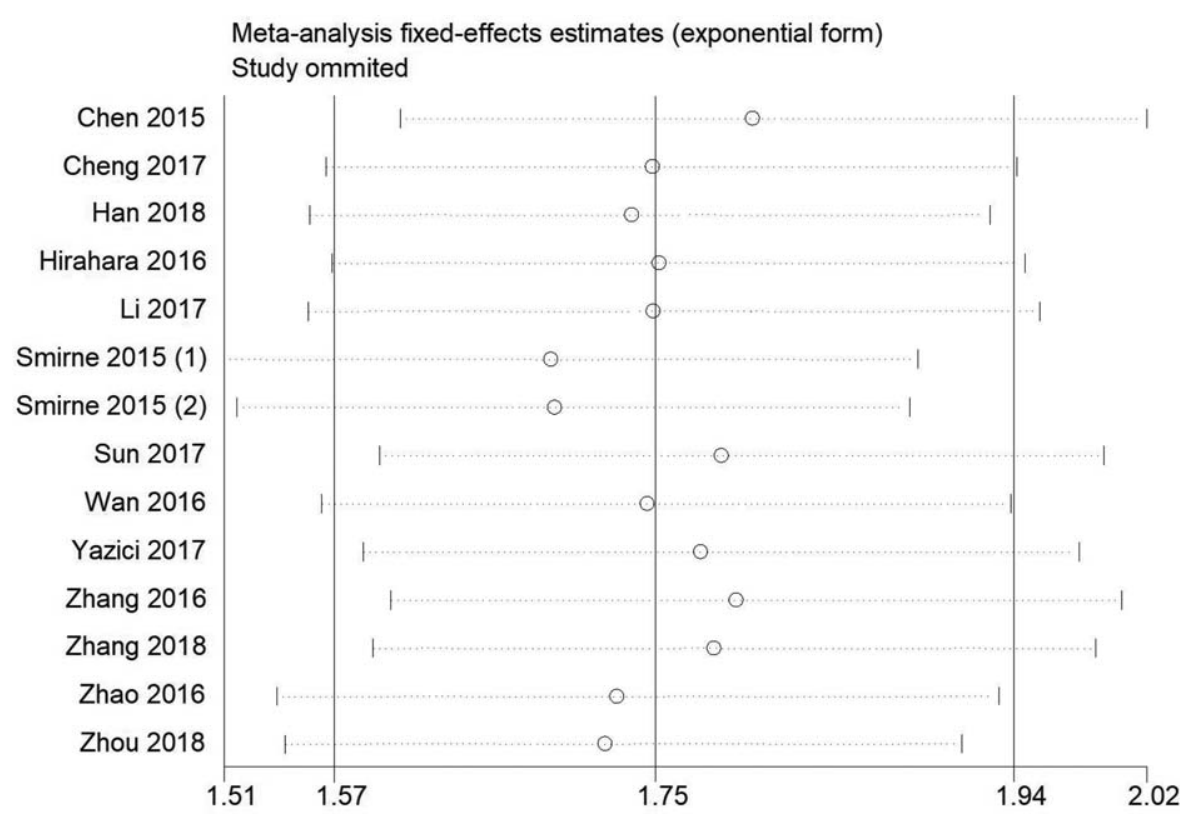

Figure 3. Sensitivity analysis for the association between RDW and OS. RDW = red blood cell distribution width, OS = overall survival. 


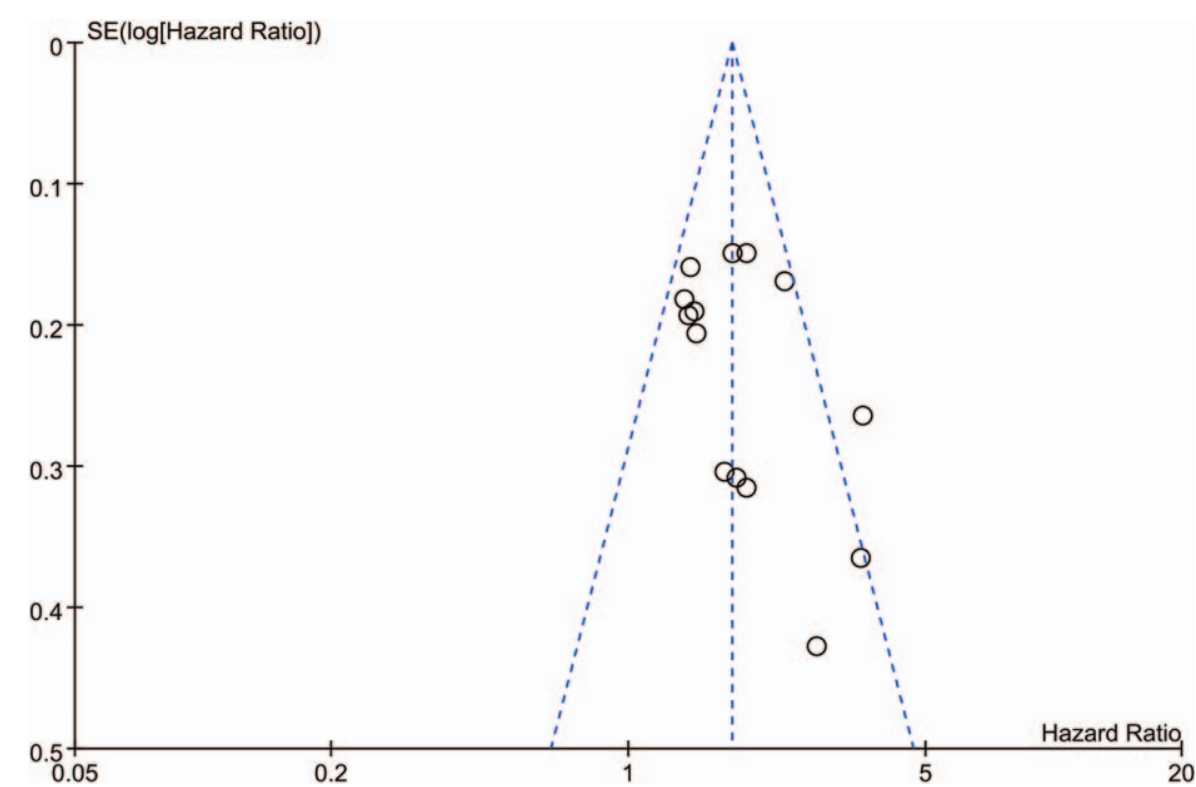

Figure 4. Funnel plot for the association between RDW and OS. RDW = red blood cell distribution width, OS = overall survival.

carcinoembryonic antigen level in GI cancers. All these findings showed RDW could serve as a promising biomarker to predict the prognosis of GI cancers.

Our results, based on the subgroup analysis, showed RDW could predict the prognosis of patients with esophageal cancer. $\mathrm{Xu}$ et al previously performed a meta-analysis, but failed to detect the relationship between RDW and prognosis in esophageal cancer, which disagreed with our findings. ${ }^{[29]}$ This difference might be on account of the contribution of $\mathrm{Hu}$ et al study, ${ }^{[30]}$ which was not included into our study because it obviously increased the heterogeneity and reduced the accuracy of results. Besides, we firstly observed the association between RDW and prognosis in gastric cancer, colorectal cancer, hepatocellular carcinoma and hilar cholangiocarcinoma in the current study. Furthermore, the subgroup analysis classified by cut-off value of RDW was conducted and the significant relationship between RDW and OS remained. However, our results suggested cut-off value of RDW was not related to the HR of OS in GI cancers.

\section{Table 2}

Subgroup analyses for the association between RDW and OS.

\begin{tabular}{|c|c|c|c|c|c|}
\hline Variables & Included studies (n) & HR 95\% Cl & $P$ & $l^{2}(\%)$ & Model \\
\hline \multicolumn{6}{|l|}{ Analysis model } \\
\hline Multivariate & 8 & $1.63[1.42,1.86]$ & $<.01^{*}$ & 0 & Fixed \\
\hline Univariate & 4 & $1.62[1.28,2.05]$ & $<.01^{*}$ & 46 & Fixed \\
\hline$<200$ & 5 & $1.84[1.50,2.24]$ & $<.01^{*}$ & 13 & Fixed \\
\hline$\geq 200$ & 7 & $1.52[1.32,1.76]$ & $<.01^{*}$ & 0 & Fixed \\
\hline \multicolumn{6}{|l|}{ Country } \\
\hline \multicolumn{6}{|c|}{ Cut-off value (\%) } \\
\hline$\leq 13$ & 2 & $1.46[1.07,1.99]$ & $.02^{*}$ & 0 & Fixed \\
\hline$\overline{13}-14$ & 3 & $2.21[1.17,4.20]$ & $.04^{*}$ & 68 & Random \\
\hline $14-15$ & 4 & $1.63[1.40,1.91]$ & $<.01^{*}$ & 0 & Fixed \\
\hline$\geq 15$ & 2 & $1.57[1.12,2.20]$ & $<.01^{*}$ & 0 & Fixed \\
\hline \multicolumn{6}{|l|}{ Type of cancer } \\
\hline HCGC & 1 & $1.76[1.31,2.35]$ & $<.01^{*}$ & NA & Fixed \\
\hline
\end{tabular}

$\mathrm{Cl}=$ confidence interval, $\mathrm{CRC}=$ colorectal cancer, $\mathrm{ES}=$ esophageal cancer, $\mathrm{GC}=$ gastric cancer, $\mathrm{HCC}=$ hepatocellular carcinoma, $\mathrm{HCGC}=$ hilar cholangiocarcinoma, $\mathrm{HR}=$ hazard ratio, NA= not available, $\mathrm{OS}=$ overall survival, $\mathrm{RDW}=$ red blood cell distribution width.

${ }^{*}$ The association was significant when $P<.05$. 


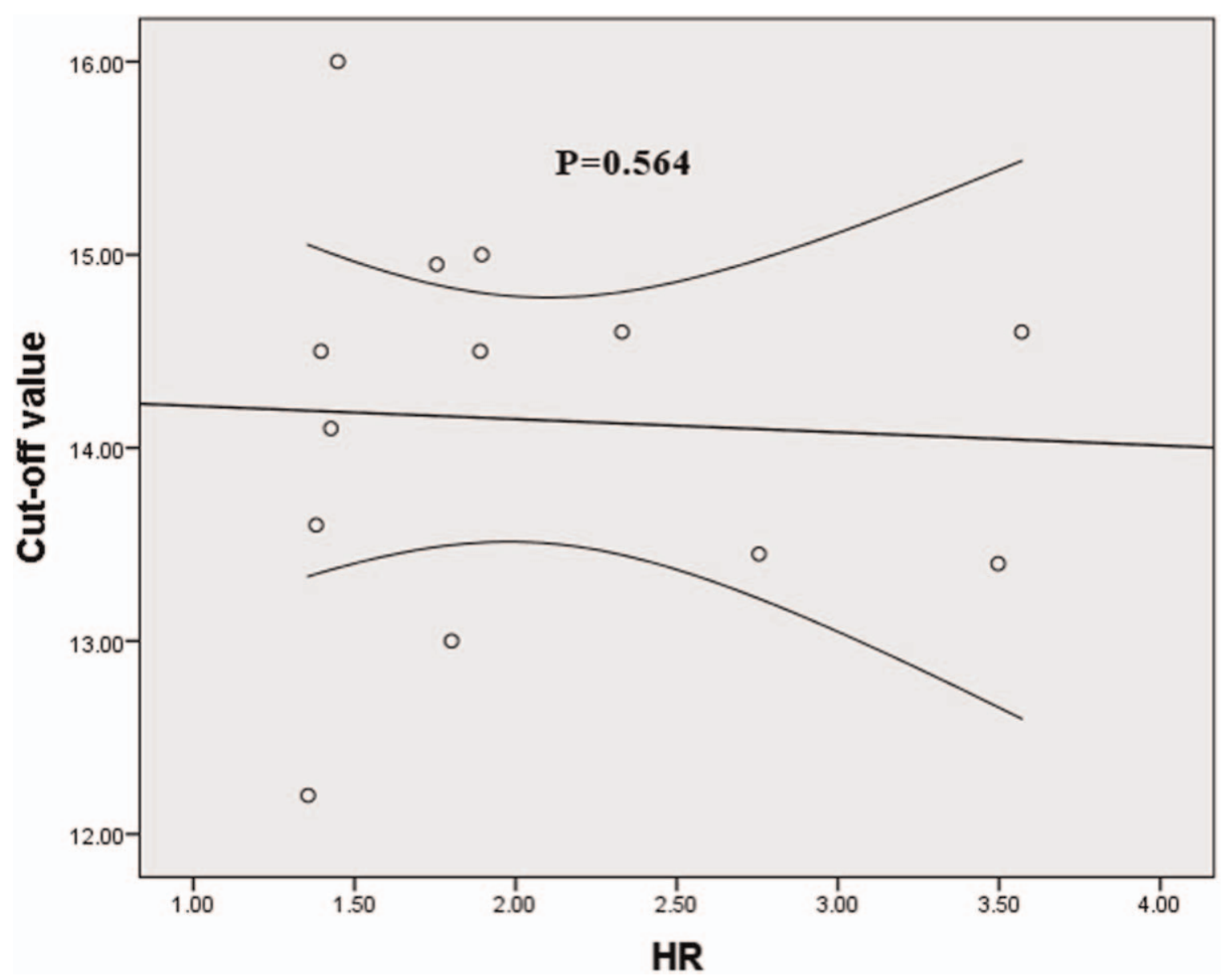

Figure 5. Correlation analysis between cut-off value of RDW and HR of OS. HR = hazad ratio, RDW = red blood cell distribution width, OS = overall survival.

Hence, researchers should pay more attention to identify the optimal cut-off value of RDW to predict the prognosis of GI cancers in future.

Although plenty of studies have explored the prognostic role of RDW in human cancers, the underlying mechanism remains vague. ${ }^{[14-25]}$ RDW may contribute to the cancer prognosis by correlating with other tumor biomarkers, such as interleukin-6, tumor necrosis factor-alpha and other cytokines. ${ }^{[31,32]}$ In addition, chronic inflammation can lead to poor response to the chemotherapy, which may induce worse prognosis of cancer patients. ${ }^{[33]}$ What's more, RDW is believed to regulate the cancer progression by affecting the glycolytic process of tumor cells, ${ }^{[34]}$ and low RDW is found to be associated with increased incidence of diabetes mellitus. ${ }^{[35]}$ Therefore, high RDW may be a surrogate indicator of improved glucose metabolism, which is of great importance for the survival of patients with GI cancers.

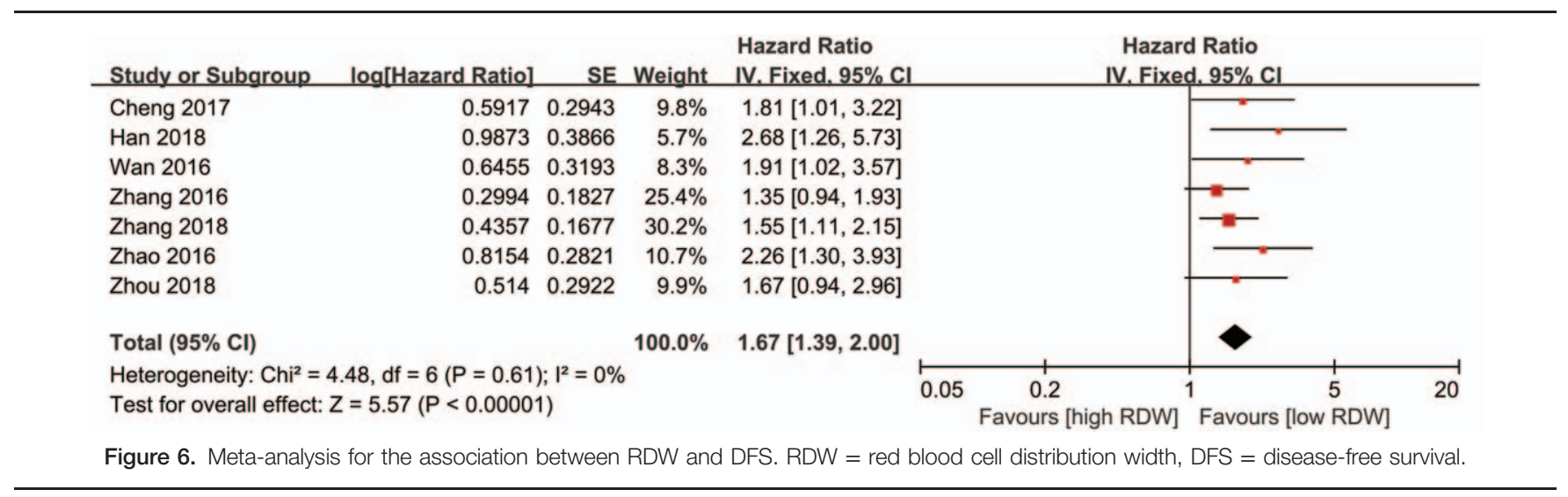




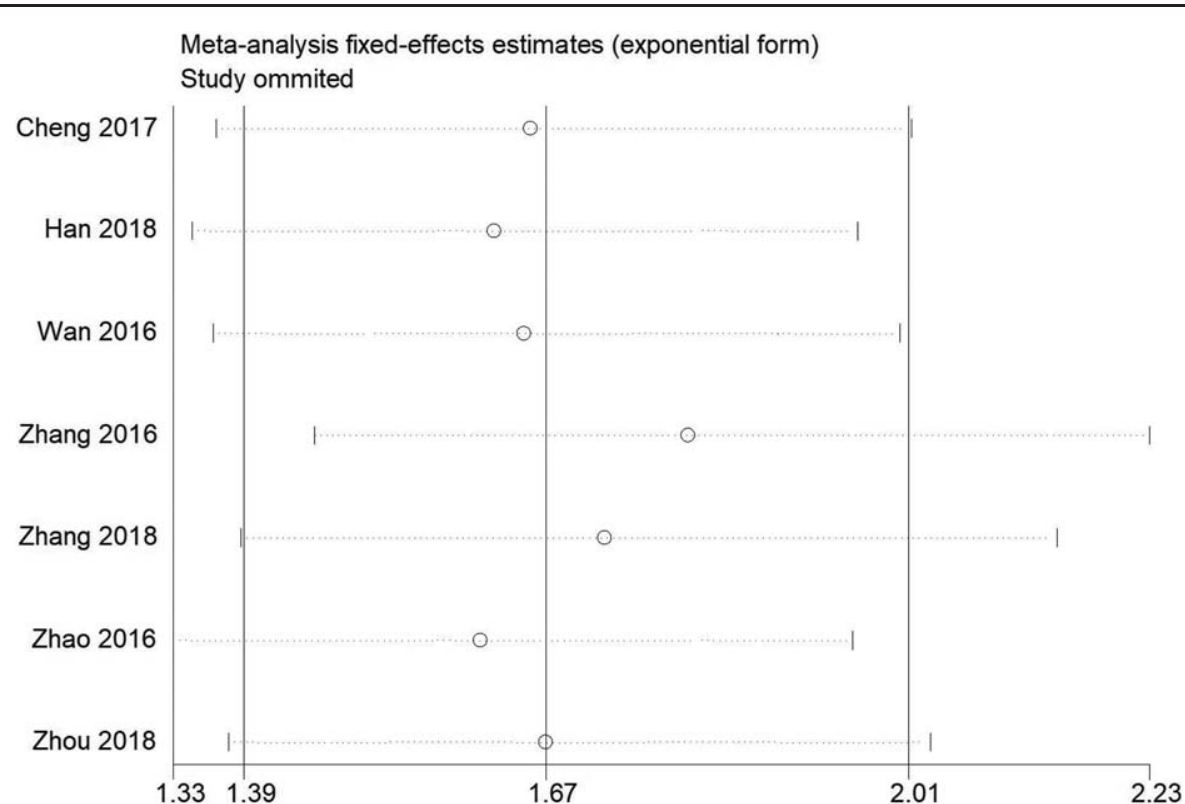

Figure 7. Sensitivity analysis for the association between RDW and DFS. RDW = red blood cell distribution width, DFS = disease-free survival.

Therefore, more preclinical experiments are warranted to testify the accurate mechanism of the prognostic significance of RDW in GI cancers.

There were several highlights of this study. First, this study was the first meta-analysis to explore the prognostic significance of RDW in GI cancers. Second, the majority of analyses were conducted without obvious heterogeneity, which guaranteed the accuracy of the results. Third, subgroup analyses were comprehensively performed in this study, which provided adequate evidence on this topic. Fourth, a total of 3,509 patients were included into this study, and the relatively large sample size could offer persuasive evidence on the prognostic value of RDW in GI cancers.

Some limitations should be considered when interpreting the results of our study. First, the sample size in specific subgroup analysis was relatively small, which failed to obtain the forceful results. However, we have tried our best to seek relevant studies, and limited relevant studies might be duo to the novelty of this topic. Second, our study failed to identify the ideal cut-off value of RDW, which might lower the clinical applicability of the conclusion. Third, many factors might affect the prognosis of patients with GI cancers, such as clinical stage at initial diagnosis,

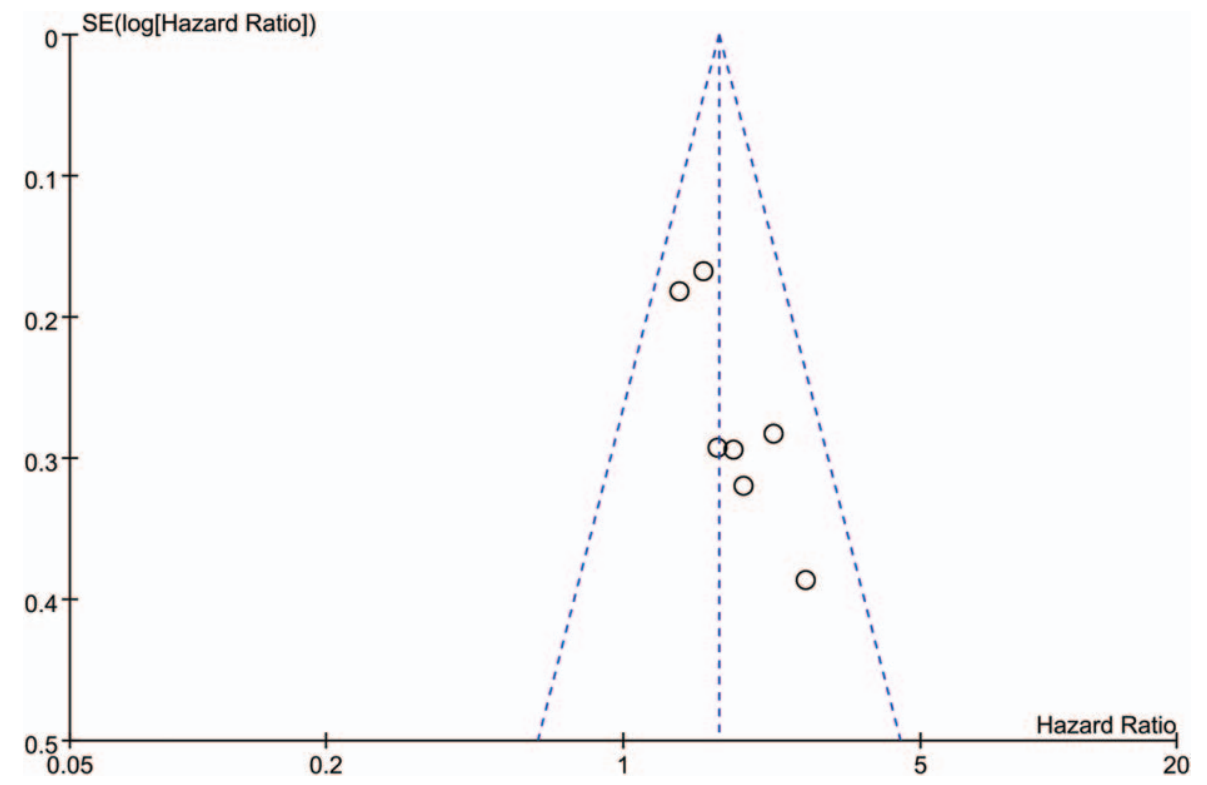

Figure 8. Funnel plot for the association between RDW and DFS. RDW = red blood cell distribution width, DFS = disease-free survival. 
Chou et al. Medicine (2020) 99:16

Medicine

Table 3

Meta-analyses for the association between RDW and clinical parameters.

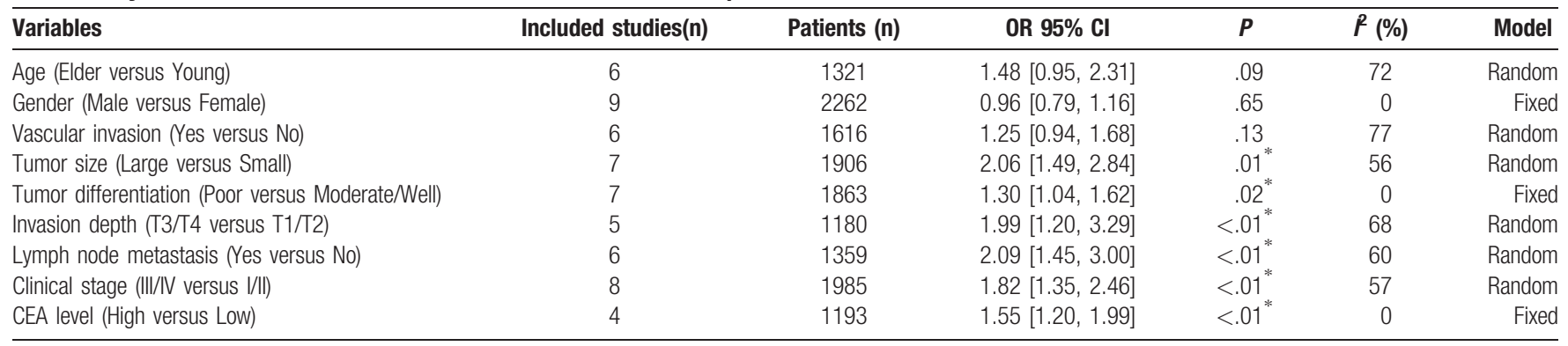

$\mathrm{CEA}=$ carcinoembryonic antigen, $\mathrm{Cl}=$ confidence interval, $\mathrm{OR}=$ odd ratio, $\mathrm{RDW}=$ red blood cell distribution width. * The association was significant when $P<.05$.

treatments, pathological types of cancer and so on. However, individual's information was unavailable for us, which was an inherent shortcoming for all meta-analyses. Forth, we explored the prognostic value of RDW in GI cancers instead of one specific cancer, which might limit the applicability of our findings. Last but not least, most included studies were conducted in China, therefore, it might be difficult to generalize the conclusion into other countries.
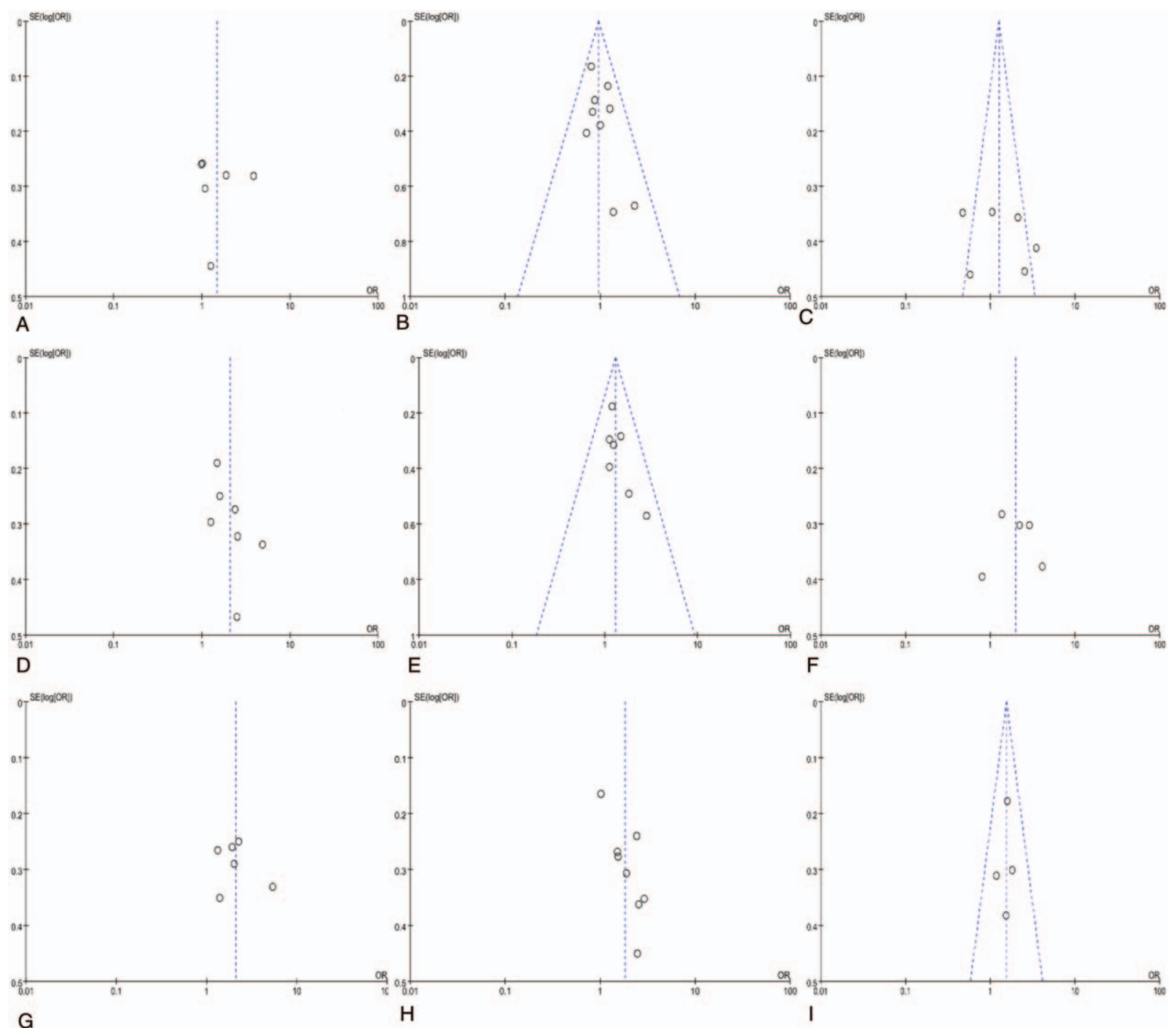

Figure 9. Funnel plots for the association between RDW and clinical parameters (a, age; b, gender; c, vascular invasion; d, tumor size; e, tumor differentiation; $f$, invasion depth; g, lymph node metastasis; h, clinical stage; i, CEA level). RDW = red blood cell distribution width.

8 


\section{Conclusion}

High RDW was significantly associated with worse prognosis when compared to low RDW in GI cancers, which could be regarded as a prognostic biomarker for GI cancers. More prospective studies with large sample size and long follow-up period should be carried out to determine the prognostic significance of RDW in GI cancers in future.

\section{Acknowledgments}

We would like to thank the researchers and study participants for their contributions.

\section{Author contributions}

Study concepts and design: Yongping Zhou and Tu Dai; Literature search: Yongping Zhou and Xiding Li; Data extraction: Yongping Zhou and Xiding Li; Statistical analysis: Yongping Zhou, Zhihua Lu and Lei Zhang; Manuscript preparation and revision: Tu Dai, Zhihua Lu and Lei Zhang. All authors have participated sufficiently in the study and approved the final version.

\section{References}

[1] Torre LA, Bray F, Siegel RL, et al. Global cancer statistics, 2012. CA Cancer J Clin 2015;65:87-108.

[2] Dupart J, Zhang W, Trent JC. Gastrointestinal stromal tumor and its targeted therapeutics. Chin J Cancer 2011;30:303-14.

[3] Acin-Gandara D, Pereira-Perez F, Castano-Pascual A, et al. Gastrointestinal stromal tumors: diagnosis and treatment. Cir Cir 2012;80:44-51.

[4] Alderson D, Winter DC. Improving outcomes in gastrointestinal cancer. Br J Surg 2013;100:1-2.

[5] Wang J, Zhao Y, Qi R, et al. Prognostic role of podocalyxin-like protein expression in various cancers: a systematic review and meta-analysis. Oncotarget 2017;8:52457-64.

[6] Zhao Y, Si G, Zhu F, et al. Prognostic role of platelet to lymphocyte ratio in hepatocellular carcinoma: a systematic review and meta-analysis. Oncotarget 2017;8:22854-62.

[7] Zhou Y, Cheng S, Chen S, et al. Prognostic and clinicopathological value of SIRT3 expression in various cancers: a systematic review and metaanalysis. Onco Targets Ther 2018;11:2157-67.

[8] Zhou Y, Cheng S, Fathy AH, et al. Prognostic value of platelet-tolymphocyte ratio in pancreatic cancer: a comprehensive meta-analysis of 17 cohort studies. Onco Targets Ther 2018;11:1899-908.

[9] Balkwill F, Mantovani A. Inflammation and cancer: back to Virchow? Lancet (London, England) 2001;357:539-45.

[10] Diakos CI, Charles KA, McMillan DC, et al. Cancer-related inflammation and treatment effectiveness. Lancet Oncol 2014;15:e493-503.

[11] Elinav E, Nowarski R, Thaiss CA, et al. Inflammation-induced cancer: crosstalk between tumours, immune cells and microorganisms. Nat Rev Cancer 2013;13:759-71.

[12] Li MX, Liu XM, Zhang XF, et al. Prognostic role of neutrophil-tolymphocyte ratio in colorectal cancer: a systematic review and metaanalysis. Int J Cancer 2014;134:2403-13.

[13] Hu RJ, Ma JY, Hu G. Lymphocyte-to-monocyte ratio in pancreatic cancer: Prognostic significance and meta-analysis. Clin Chim Acta 2018;481:142-6.

[14] Chen GP, Huang Y, Yang X, et al. A nomogram to predict prognostic value of red cell distribution width in patients with esophageal cancer. Mediators Inflamm 2015;2015:854670.
[15] Cheng S, Han F, Wang Y, et al. The red distribution width and the platelet distribution width as prognostic predictors in gastric cancer. BMC Gastroenterol 2017;17:163.

[16] Hirahara N, Matsubara T, Kawahara D, et al. Prognostic value of hematological parameters in patients undergoing esophagectomy for esophageal squamous cell carcinoma. Int J Clin Oncol 2016;21:909-19.

[17] Li B, You Z, Xiong XZ, et al. Elevated red blood cell distribution width predicts poor prognosis in hilar cholangiocarcinoma. Oncotarget 2017;8:109468-77.

[18] Smirne C, Grossi G, Pinato DJ, et al. Evaluation of the red cell distribution width as a biomarker of early mortality in hepatocellular carcinoma. Dig Liver Dis 2015;47:488-94.

[19] Sun P, Zhang F, Chen C, et al. The ratio of hemoglobin to red cell distribution width as a novel prognostic parameter in esophageal squamous cell carcinoma: a retrospective study from southern China. Oncotarget 2016;7:42650-60.

[20] Wan GX, Chen P, Cai XJ, et al. Elevated red cell distribution width contributes to a poor prognosis in patients with esophageal carcinoma. Clin Chim Acta 2016;452:199-203.

[21] Yazici P, Demir U, Bozkurt E, et al. The role of red cell distribution width in the prognosis of patients with gastric cancer. Cancer Biomark 2017;18:19-25.

[22] Zhang F, Chen Z, Wang P, et al. Combination of platelet count and mean platelet volume (COP-MPV) predicts postoperative prognosis in both resectable early and advanced stage esophageal squamous cell cancer patients. Tumour Biol 2016;37:9323-31.

[23] Zhang X, Wu Q, Hu T, et al. Elevated red blood cell distribution width contributes to poor prognosis in patients undergoing resection for nonmetastatic rectal cancer. Medicine 2018;97:e9641.

[24] Zhao T, Cui L, Li A. The significance of RDW in patients with hepatocellular carcinoma after radical resection. Cancer Biomark 2016;16:507-12.

[25] Zhou D, Wu Y, Lin Z, et al. Prognostic value of combination of pretreatment red cell distribution width and neutrophil-to-lymphocyte ratio in patients with gastric cancer. Gastroenterol Res Pract 2018; 2018:8042838.

[26] Han F, Shang X, Wan F, et al. Clinical value of the preoperative neutrophil-to-lymphocyte ratio and red blood cell distribution width in patients with colorectal carcinoma. Oncol Lett 2018;15:3339-49.

[27] Tierney JF, Stewart LA, Ghersi D, et al. Practical methods for incorporating summary time-to-event data into meta-analysis. Trials 2007;8:16.

[28] Stang A. Critical evaluation of the Newcastle-Ottawa scale for the assessment of the quality of nonrandomized studies in meta-analyses. Eur J Epidemiol 2010;25:603-5.

[29] Xu WY, Yang XB, Wang WQ, et al. Prognostic impact of the red cell distribution width in esophageal cancer patients: a systematic review and meta-analysis. World J Gastroenterol 2018;24:2120-9.

[30] Hu D, Lin X, Chen Y, et al. Preoperative blood-routine markers and prognosis of esophageal squamous cell carcinoma: the Fujian prospective investigation of cancer (FIESTA) study. Oncotarget 2017; 8:23841-50.

[31] Rhodes CJ, Howard LS, Busbridge M, et al. Iron deficiency and raised hepcidin in idiopathic pulmonary arterial hypertension: clinical prevalence, outcomes, and mechanistic insights. J Am Coll Cardiol 2011;58: 300-9.

[32] Lippi G, Targher G, Montagnana M, et al. Relation between red blood cell distribution width and inflammatory biomarkers in a large cohort of unselected outpatients. Arch Pathol Lab Med 2009;133:628-32.

[33] Balkwill FR, Mantovani A. Cancer-related inflammation: common themes and therapeutic opportunities. Semin Cancer Biol 2012;22: 33-40.

[34] Gillies RJ, Robey I, Gatenby RA. Causes and consequences of increased glucose metabolism of cancers. J Nucl Med 2008;49 Suppl 2:24s-42s.

[35] Engstrom G, Smith JG, Persson M, et al. Red cell distribution width, haemoglobin A1c and incidence of diabetes mellitus. J Intern Med 2014;276:174-83. 